EPJ Web of Conferences 37, 09018 (2012)

DOI: $10.1051 /$ epjconf/20123709018

(C) Owned by the authors, published by EDP Sciences, 2012

\title{
Formation of deeply bound pionic atoms in Sn isotopes
}

\author{
Natsumi Ikeno ${ }^{1, a}$, Hideko Nagahiro ${ }^{1}$, Daisuke $\mathrm{Jido}^{2}$, and Satoru Hirenzaki ${ }^{1}$ \\ 1 Department of Physics, Nara Women's University, Nara 630-8506, Japan \\ 2 Yukawa Institute for Theoretical Physics, Kyoto University, Kyoto 606-8502, Japan
}

\begin{abstract}
We study the formation of deeply bound pionic atoms in the $\left(d,{ }^{3} \mathrm{He}\right)$ reactions theoretically and show the energy spectra of the emitted ${ }^{3} \mathrm{He}$ at finite angles. We find that the different combinations of the pion-bound and neutron-hole states dominate the formation spectra at different scattering angles because of the matching condition of the reaction. We conclude that the observation of the $\left(d,{ }^{3} \mathrm{He}\right)$ reaction at finite angles will provide the systematic information of the pionic bound states in each nucleus, and it will help to develop the study of the pion properties and the partial restoration of chiral symmetry in nuclei.
\end{abstract}

\section{Introduction}

Deeply bound pionic atom is one of the best systems to deduce pion properties at finite density and to obtain precise information on partial restoration of chiral symmetry in nuclei [1].

So far, the deeply bound pionic states have been experimentally produced in the forward $\left(d,{ }^{3} \mathrm{He}\right)$ reaction. The $\left(d,{ }^{3} \mathrm{He}\right)$ spectra have been obtained in near recoilless kinematics to observe the peaks of the pionic state formation with a neutron hole in the quasi-substitutional configurations [2-4]. The $\mathrm{Pb}$ and $\mathrm{Sn}$ isotopes were used as the target nuclei in the experiments [2-4] since the spectra were expected to show the peak structures owing to one dominant $\left[\pi \otimes n^{-1}\right]$ configuration based on the theoretical evaluations [5,6]. The simple structure of the observed peak is important and effective to deduce the pion binding energies precisely from the observed spectra. Actually, in Ref. [3], the binding energy and width of the 1s states have been precisely measured in three $\mathrm{Sn}$ isotopes and isospin-density dependence of the s-wave pion-nucleus potential has been deduced. From these observations, the reduction of the chiral order parameter $\langle\bar{q} q\rangle$ in nucleus was concluded. A recent model independent theoretical analysis supported the way to extract the in-medium quark condensate from the pionic atom data and showed a relation connecting the in-medium quark condensate to the hadronic observables [7].

To develop the studies of pion properties and symmetry restoration in nuclei further, we need to obtain precise and systematic information on deeply bound pionic states. For example, we need more systematic information on the bound states for the unique determination of the pion-nucleus interaction, which is required to fix the potential strength related to chiral symmetry [8]. Recently, it was reported in Ref. [8] that the simultaneous observation of various pionic bound states such as $1 s$ and $2 s$ in the same nucleus may be helpful to reduce these errors and to develop our studies. Along with this line, in actual experiments, the pionic $2 s$ state observation with the $1 s$ state has been expected in new high precision experiment in RIBF/RIKEN [8-10].

In this paper, we consider theoretically the new possibility to observe various pionic bound states in the same nuclei by observing the $\left(d,{ }^{3} \mathrm{He}\right)$ spectra at finite angles together with the forward direction. These observations at finite angles were reported by the experiments at RIBF/RIKEN [11]. At finite angle, we can expect to have the manifestation of different subcomponents of pion and neutron hole

\footnotetext{
a e-mail: jan_ikeno@cc.nara-wu.ac.jp
}

This is an Open Access article distributed under the terms of the Creative Commons Attribution License 2.0, which permits unrestricted use, distribution, and reproduction in any medium, provided the original work is properly cited. 
states due to the matching condition with different momentum transfer, and expect to determine the binding energies and widths of various pionic states simultaneously in each nucleus.

\section{Formulation}

We use the effective number approach to calculate the pionic atom formation cross sections [12]. We refine the theoretical model used in Refs. $[5,6,13]$ to study the angular dependence of the $\left(d,{ }^{3} \mathrm{He}\right)$ spectra by including the kinematical correction factors $K$ in Eq. (1) as explained below. The $\left(d,{ }^{3} \mathrm{He}\right)$ reaction cross section in the laboratory frame is expressed as,

$$
\left(\frac{d^{2} \sigma}{d E_{\mathrm{He}} d \Omega_{\mathrm{He}}}\right)_{A}^{\mathrm{lab}}=\left(\frac{d \sigma}{d \Omega_{\mathrm{He}}}\right)_{\mathrm{ele}}^{\mathrm{lab}} \sum_{p h} K \frac{\Gamma}{2 \pi} \frac{1}{\Delta E^{2}+\Gamma^{2} / 4} N_{\mathrm{eff}}
$$

where $\left(\frac{d \sigma}{d \Omega_{\mathrm{He}}}\right)_{\text {ele }}^{\mathrm{lab}}$ indicates the elementary cross section for the $d+n \rightarrow{ }^{3} \mathrm{He}+\pi^{-}$reaction in lab frame. As the elementary cross section, we have used the experimental data of $p+d \rightarrow t+\pi^{+}$reaction reported in Ref [14] in the CM frame by isospin symmetry.

The effective number $N_{\text {eff }}$ defined as,

$$
N_{\mathrm{eff}}=\sum_{J M}\left|\int d \mathbf{r} \chi_{\mathrm{He}}^{*}(\mathbf{r})\left[\phi_{\ell_{\pi}}^{*}(\mathbf{r}) \otimes \psi_{j_{n}}(\mathbf{r})\right]_{J M} \chi_{d}(\mathbf{r})\right|^{2},
$$

where $\phi_{\ell_{\pi}}$ and $\psi_{j_{n}}$ indicate the wavefunctions of the pion bound state in the daughter nucleus and the neutron bound state in the target nucleus, respectively. For the neutron wave function $\psi_{j_{n}}$, we use the calculated wave function using the neutron potential reported in Ref. [15]. The wave functions of the projectile $(d)$ and the ejectile $\left({ }^{3} \mathrm{He}\right)$ are denoted by $\chi_{\mathrm{He}}^{*}$ and $\chi_{d}$. We introduce the distortion effects to the wavefunctions $\chi_{\mathrm{He}}^{*}$ and $\chi_{d}$ by Eikonal approximation as described in Refs. [5,6,13].

The kinematical correction factor $K$ is defined as,

$$
K=\left[\frac{\left|\mathbf{p}_{\mathrm{He}}^{A}\right|}{\left|\mathbf{p}_{\mathrm{He}}\right|} \frac{E_{n} E_{\pi}}{E_{n}^{A} E_{\pi}^{A}}\left(1+\frac{E_{\mathrm{He}}}{E_{\pi}} \frac{\left|\mathbf{p}_{\mathrm{He}}\right|-\left|\mathbf{p}_{d}\right| \cos \theta_{d \mathrm{He}}}{\left|\mathbf{p}_{\mathrm{He}}\right|}\right)\right]^{\mathrm{lab}},
$$

where $A$ indicates the momentum and energy which should be evaluated in the kinematics of the nuclear target case. The superscript 'lab' indicates that all kinematical variables are evaluated in the laboratory frame. This correction factor is $K=1$ for the recoilless kinematics at $\theta_{d \mathrm{He}}^{\text {lab }}=0^{\circ}$ with $S_{n}=0$ and B.E. $=0$. In Ref. [12], we find that the $K$ factor gradually varies about $10 \%$ within the $Q$-value range considered here for both $\theta_{d \mathrm{He}}^{\mathrm{lab}}=0^{\circ}$ and $2^{\circ}$ cases, and the $K$ factor decreases about $20 \%$ at $\theta_{d \mathrm{He}}^{\mathrm{lab}}=2^{\circ}$ from the value at $\theta_{d \mathrm{He}}^{\mathrm{lab}}=0^{\circ}$. Thus, we think the $K$ factor should be introduced to study the angular dependence of the $\left(d,{ }^{3} \mathrm{He}\right)$ spectra.

\section{Numerical Results and Discussions}

In Fig. 1, we show the calculated $\left(d,{ }^{3} \mathrm{He}\right)$ spectra at finite angles for the bound pionic states formation. We find that the spectra have a strong angular dependence and the shape of the spectra are much different at finite angles from that at $0^{\circ}$. The largest peak structure at $Q=-137.8 \mathrm{MeV}$ in the forward spectra is strongly suppressed at finite angles and the spectra show the structure of three peaks at $\theta_{d \mathrm{He}}^{\mathrm{lab}} \geq 2^{\circ}$. The overall strength of the spectra has also angular dependence and is smaller for larger angles.

In Fig. 2, we show the dominant subcomponents of the $\left(d,{ }^{3} \mathrm{He}\right)$ spectra for each scattering angle $\theta_{d \mathrm{He}}^{\mathrm{lab}}$. At $\theta_{d \mathrm{He}}^{\mathrm{lab}}=0^{\circ}$, since the reaction is close to recoilless, the peak of the $\left[(1 s)_{\pi} \otimes\left(3 s_{1 / 2}\right)_{n}^{-1}\right]$ and 


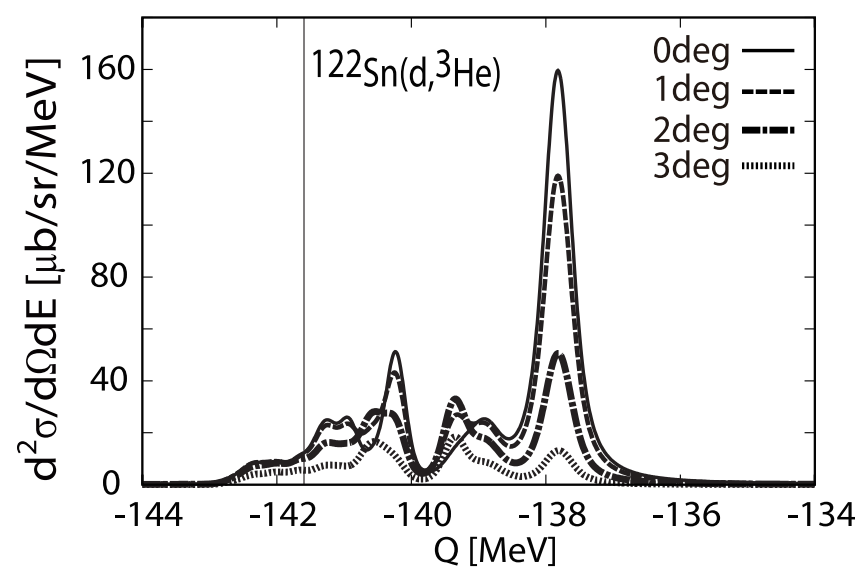

Fig. 1. Calculated ${ }^{122} \mathrm{Sn}\left(d,{ }^{3} \mathrm{He}\right)$ spectra for the formation of the pionic bound states at $\theta_{d \mathrm{He}}^{\text {lab }}=0^{\circ}$ (solid lines), $1^{\circ}$ (dash lines), $2^{\circ}$ (dash-dotted lines) and $3^{\circ}$ (dotted lines) plotted as functions of the reaction $Q$-value [12]. The incident deuteron kinetic energy is fixed to be $T_{d}=500 \mathrm{MeV}$. The instrumental energy resolution is assumed to be $300 \mathrm{keV}$ FWHM. The vertical line indicates the pion production threshold $Q=-141.6 \mathrm{MeV}$.

$\left[(2 s)_{\pi} \otimes\left(3 s_{1 / 2}\right)_{n}^{-1}\right]$ subcomponents appear clearly in the spectra. At $\theta_{d \mathrm{He}}^{\text {lab }}=1^{\circ}$, the contribution for $\left[(2 s)_{\pi} \otimes\left(3 s_{1 / 2}\right)_{n}^{-1}\right]$ is suppressed and can only be seen clearly if the energy resolution is better than 300 $\mathrm{keV}$. At larger angles, the pionic $(2 p)$ state contributions become relatively larger and dominate the peak structure around $Q=-139.4 \mathrm{MeV}$ and $-140.4 \mathrm{MeV}$. We can expect to observe the peak structure composed from the $\left[(2 p)_{\pi} \otimes\left(3 s_{1 / 2}\right)_{n}^{-1}\right],\left[(2 p)_{\pi} \otimes\left(2 d_{3 / 2}\right)_{n}^{-1}\right]$ and $\left[(2 p)_{\pi} \otimes\left(1 h_{11 / 2}\right)_{n}^{-1}\right]$ subcomponents. Though, the separation energies of these 3 neutron levels differ from each other only within $60 \mathrm{keV} \mathrm{[8]}$ and their contributions can not be distinguished, we can expect to deduce the information on the pionic $2 p$ state. These contributions of pionic $2 p$ state can not be seen in the spectra at $\theta_{d \mathrm{He}}^{\mathrm{lab}}=0^{\circ}$ since they are hidden in the tail of the large $1 s$ contributions. At finite angles, due to the significant suppression of the $1 s$ contributions, the $2 p$ contributions can be observed even if they are a little smaller than those at the forward angle. Thus, to observe the spectra at finite angle is valuable.

\section{Summary}

We study the formation of deeply bound pionic atoms in the $\left(d,{ }^{3} \mathrm{He}\right)$ reactions theoretically. We develop the formula to include the kinematical correction factors to the effective number approach to obtain more realistic angular dependence of the $\left(d,{ }^{3} \mathrm{He}\right)$ spectra. We show the angular dependence of the ${ }^{122} \mathrm{Sn}\left(d,{ }^{3} \mathrm{He}\right)$ spectra at $T_{d}=500 \mathrm{MeV}$ for the formation of the pionic atoms.

We find that the spectra are dominated by the subcomponents including the $(2 p)_{\pi}$ state at larger scattering angles $\theta_{d \mathrm{He}}^{\mathrm{lab}} \geq 2^{\circ}$, while they are dominated by the $(1 s)_{\pi}$ and $(2 s)_{\pi}$ states at forward angles. The peaks are well isolated and can be observed in the experiments with the good energy resolution. Thus, we can conclude that we can obtain information on deeply bound pionic $2 p$ state in addition to $1 s$ and $2 s$ states by observing the spectra at finite angles. As indicated in Ref. [8], the observation of several deeply pionic bound states in a certain nucleus will help to deduce precise information of pion properties and chiral dynamics at finite density $[3,7,16]$. We believe that our results provide a good evaluation for further experimental studies of the states reported here, which should contribute to the development of the field.

N. I. appreciates the support by the Grant-in-Aid for JSPS Fellows (No. 23·2274).

\section{References}

1. For review: T. Yamazaki, S. Hirenzaki, R.S. Hayano, H. Toki, Phys. Rep. 514, (2012) 1. 

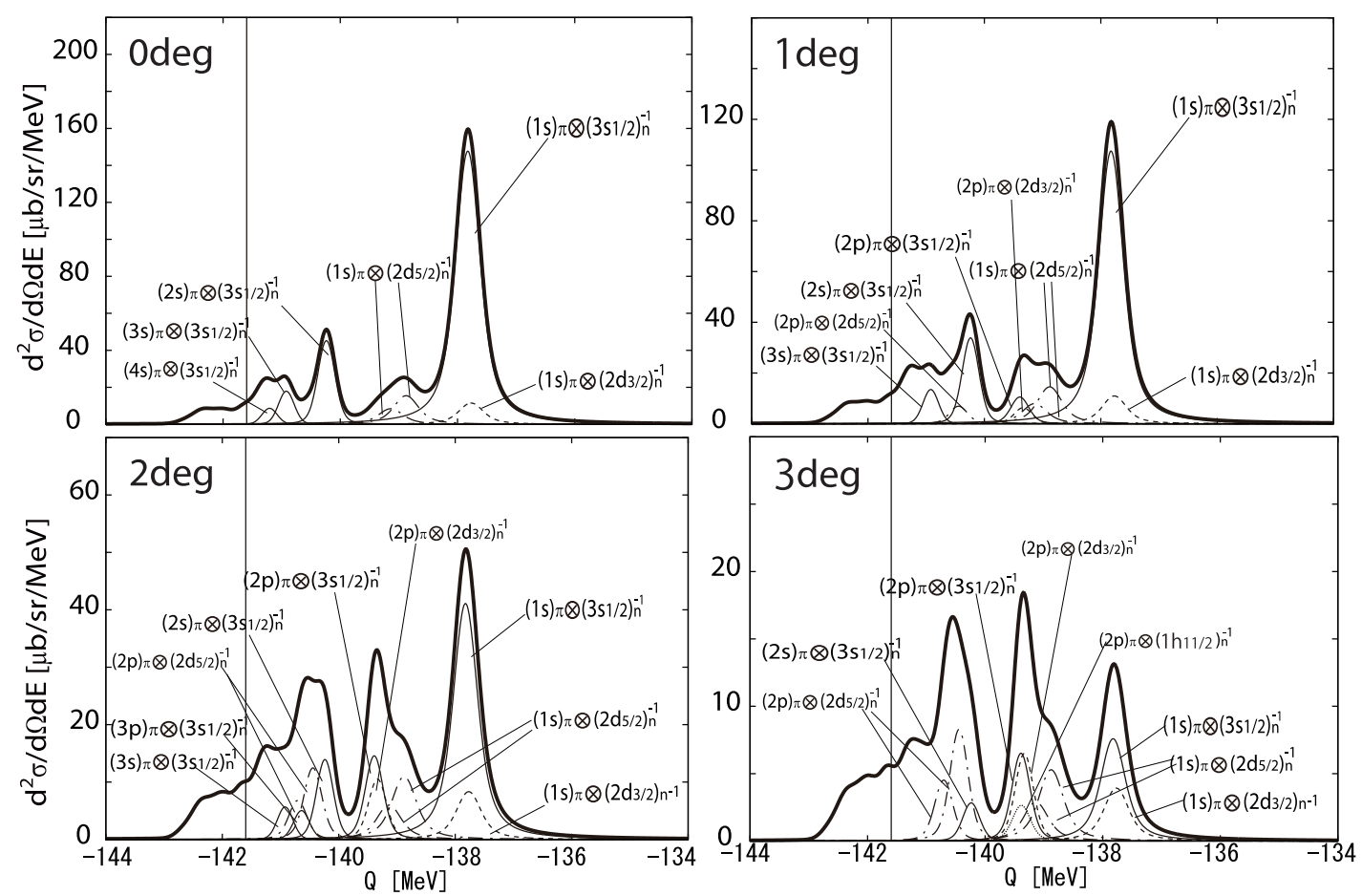

Fig. 2. Calculated ${ }^{122} \mathrm{Sn}\left(d,{ }^{3} \mathrm{He}\right)$ spectra for the formation of the pionic bound states at $\theta_{d \mathrm{He}}^{\mathrm{lab}}=0^{\circ}, 1^{\circ}, 2^{\circ}$ and $3^{\circ}$ are plotted as functions of the reaction $Q$-value [12]. Dominant subcomponents $\left[(n \ell)_{\pi} \otimes\left(n \ell_{j}\right)_{n}^{-1}\right]$ are indicated in the figure. The instrumental energy resolution is assumed to be $300 \mathrm{keV}$ FWHM. The vertical line indicates the pion production threshold $Q=-141.6 \mathrm{MeV}$.

2. K. Itahashi et al., Phys. Rev. C62, (2000) 025202.

3. K. Suzuki et al., Phys. Rev. Lett. 92, (2004) 072302.

4. H. Geissel et al., Phys. Lett. B549, (2002) 64.

5. Y. Umemoto, S. Hirenzaki, K. Kume and H. Toki, Phys. Rev. C62, (2000) 024606.

6. Y. Umemoto, S. Hirenzaki, K. Kume and H. Toki, Prog. Theor. Phys. 103, (2000) 337.

7. D. Jido, T. Hatsuda and T. Kunihiro, Phys. Lett. B670, (2008) 109; see also S. Goda, D. Jido, in these proceedings.

8. N. Ikeno, R. Kimura, J. Yamagata-Sekihara, H. Nagahiro, D. Jido, K. Itahashi, L. S. Geng and S. Hirenzaki, Prog. Theor. Phys. 126, (2011) 483.

9. K. Itahashi et al., 'Precision Spectroscopy of Pionic Atoms in $\left(d,{ }^{3} \mathrm{He}\right)$ Nuclear Reactions'; Exp. proposal NP0702-RIBF-027 for RIBF, Dec. (2006).

10. K. Itahashi et al., 'Spectroscopy of Pionic Atom in ${ }^{122} \mathrm{Sn}\left(d,{ }^{3} \mathrm{He}\right)$ Nuclear Reaction'; Exp. proposal NP0802-RIBF-054 for RIBF, Jan. (2008).

11. K. Itahashi et al., in these proceedings; S. Itoh, "Precision spectroscopy of deeply bound states in the pionic ${ }^{121} \mathrm{Sn}$ atom", Doctor Thesis, University of Tokyo, December (2011).

12. N. Ikeno, H. Nagahiro and S. Hirenzaki, Eur. Phys. J. A47 (2011) 161.

13. S. Hirenzaki, H. Toki and T. Yamazaki, Phys. Rev. C44, (1991) 2472; H. Toki, S. Hirenzaki and

T. Yamazaki, Nucl. Phys. A530, (1991) 679.

14. M. Betigeri et al., Nucl. Phys. A690, (2001) 473.

15. H. Koura and M. Yamada, Nucl. Phys. A671, (2000) 96.

16. E. E. Kolomeitsev, N. Kaiser and W. Weise, Phys. Rev. Lett. 90, (2003) 092501. 